\title{
Estudo comparativo entre dois protocolos anestésicos envolvendo bloqueio do nervo alveolar inferior convencional e de Vazirani-Akinosi para exodontia de terceiro molar inferior
}

\author{
Comparative study of two anaesthetic protocols involving conventional and Vazirani-Akinosi alveolar \\ inferior nerve block for lower third molar extraction
}

Danilo de Paula Ribeiro BORGES ${ }^{a}$, Liane Maciel de Almeida SOUZA ${ }^{a}$, Maria Luisa Silveira SOUTO Liliane Poconé DANTAS ${ }^{\mathrm{a}}$, Mônica Silveira PAIXÃO ${ }^{\mathrm{a}}$, Francisco Carlos GROPPO ${ }^{\mathrm{b}}$

${ }^{a} U F S$ - Universidade Federal de Sergipe, Aracaju, SE, Brasil

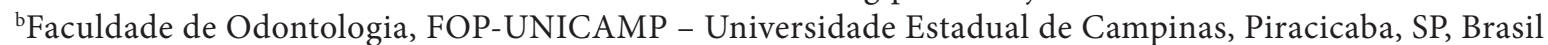

\begin{abstract}
Resumo
Introdução: O bloqueio do nervo alveolar inferior (BNAI) apresenta alta porcentagem de falha na Odontologia. A fim de melhorar esse índice, vêm-se estudando diferentes alternativas, como diferentes técnicas e soluções anestésicas. Objetivo: Avaliar duas diferentes técnicas - técnica convencional e de Vazirani-Akinosi - para o bloqueio do nervo alveolar inferior, bem como compará-las quanto à sua efetividade e quantificar o percentual de aspirações positivas nas duas diferentes técnicas. Material e método: Foram avaliados 160 pacientes de ambos os sexos, sendo 80 submetidos ao bloqueio do nervo alveolar inferior de Vazirani-Akinosi e bloqueio do nervo bucal (G1), e 80 submetidos ao bloqueio do nervo alveolar inferior convencional e ao bloqueio do nervo bucal (G2). Em ambos os grupos, utilizou-se a combinação de articaína 4\% com epinefrina 1:100.000 para bloqueio do nervo bucal, e lidocaína 2\% com epinefrina 1:100.000 para bloqueio do nervo alveolar inferior. Foram avaliados: a quantidade de aspirações positivas, a eficácia da anestesia e o momento em que ocorreu a falha anestésica durante o procedimento cirúrgico. Resultado: Não houve diferenças estatisticamente significantes ( $\mathrm{p}=0,2453)$ entre os grupos G1 e G2 observando-se a eficácia e o índice de aspirações positivas, e o momento em que ocorreu a falha anestésica, observando-se uma maior eficácia de ambas as técnicas, quando comparadas com a literatura (90\%) Conclusão: Não houve diferença significativa entre o BNAI pela técnica convencional e o BNAI pela técnica de Vazirani-Akinosi quanto a quantidade de aspirações positivas e eficácia, sendo que o uso da articaína $4 \%$ com epinefrina 1:100.000 no bloqueio do nervo bucal possivelmente aumentou a eficácia anestésica de ambas as técnicas.
\end{abstract}

Descritores: Articaína; nervo alveolar inferior; anestesia; cirurgia bucal.

\begin{abstract}
Introduction: The inferior alveolar nerve block has a high percentage of failure in dentistry. To improve this ratio, has been studied different alternatives, as different techniques, as well as anesthetics. Objective: Evaluate two different techniques (conventional technique and Vazirani-Akinosi) for inferior alveolar nerve block, and compare them regarding their effectiveness and quantify the percentage of positive aspirations in both techniques. Material and method: 160 patients were evaluated for both sex, with 80 undergoing Vazirani-Akinosi technique plus buccal nerve block (G1), and 80 to conventional inferior alveolar nerve block plus buccal nerve block (G2), both groups using a combination of $4 \%$ articaine with 1:100.000 epinephrine to buccal nerve block and $2 \%$ lidocaine with 1:100.000 epinephrine to inferior alveolar nerve block. We evaluated the amount of positive aspirations, the effectiveness or not of anesthesia (pain) and when it occurred during the surgical procedure. Result: There were no statistically significant differences $(\mathrm{p}=0.2453)$ between $\mathrm{G} 1$ and G2 observing the positive aspiration. It was obtained efficiency of $90 \%$ for both techniques. Conclusion: No significant difference between the conventional alveolar inferior nerve block technique and Vazirani-Akinosi alveolar nerve block technique considering the amount of positive aspirations and efficacy, and the use of $4 \%$ articaine with 1:100.000 epinephrine in buccal nerve block possibly increased the anesthetic efficacy of both techniques.
\end{abstract}

Descriptors: Carticaine; mandibular nerve; anesthesia; surgery, oral. 


\section{INTRODUÇÃO}

Na Odontologia, várias são as técnicas utilizadas para o controle da dor no manejo de pacientes que se submetem à cirurgia para extração dos terceiros molares inferiores, especialmente quando é feito o bloqueio do nervo alveolar inferior (BNAI). Destacam-se, entre estas, as técnicas convencional e de Vazirani-Akinosi ${ }^{1}$.

Ainda que haja diferentes opções de técnicas, a incidência de falhas ainda é alta. O BNAI é a técnica de injeção mais usada e a que possui maior porcentagem de fracassos clínicos (aproximadamente $15 \%$ a 20\%), mesmo quando corretamente administrada $^{2}$. A justificativa para tamanha taxa de falha advém de diversos motivos: ocorrência de nervo acessório, variação no curso do nervo alveolar inferior, variação na posição do forame e/ou nervo alveolar bífido com canal da mandíbula bífido. Outros fatores externos podem contribuir para falha anestésica, como a história dental e a severidade e a extensão da inflamação tecidual nos diversos pacientes ${ }^{3}$.

Outra falha importante durante o BNAI é a injeção intravascular de anestésico. Para Kuster, Udin ${ }^{4}$, o BNAI é o que apresenta maior frequência de aspirações positivas dentre as diversas técnicas de anestesia bucal. As consequências da injeção intravascular vão depender da quantidade de anestésico injetado e da capacidade do organismo de tolerar a droga, metabolizá-la e excretá-la ${ }^{5}$. As complicações vão de brandas a até mesmo fatais. Podem ocorrer vômito, taquicardia, palpitações, desmaio e consequente parada cardíaca $^{6}$. Para que isso não ocorra, um procedimento já consagrado na literatura é a técnica de aspiração prévia, a fim de verificar se a agulha situa-se dentro de um vaso sanguíneo. Quando da aspiração de sangue, diz-se que a aspiração foi positiva. O caso contrário é chamado de aspiração negativa, que é o objetivo durante a infiltração do anestésico ${ }^{7}$.

Para aumentar a eficácia e diminuir a quantidade de falhas da anestesia, podem-se associar diferentes técnicas e soluções anestésicas no intuito de aumentar a probabilidade de efetividade pulpar. O BNAI pela técnica convencional já é amplamente utilizado na clínica, pela sua simplicidade na aplicação e por possuir um relativo poder assertivo. Outra alternativa, a técnica de Vazirani-Akinosi, foi desenvolvida em 1977, a fim de possibilitar o bloqueio do nervo alveolar inferior de pacientes que não podiam abrir totalmente a boca. Desde então, esta técnica se tornou uma alternativa bastante viável, devido à sua facilidade, já que não necessita de pontos de referência extraorais, proporcionando uma menor sensação dolorosa relatada pelo paciente durante a punção, além de uma taxa reduzida de aspirações positivas, pelo fato de que há um menor número de vasos sanguíneos localizados na zona de punção ${ }^{8}$. A técnica convencional, se comparada à de Vazirani-Akinosi, tem uma eficácia clínica semelhante, porém torna-se inviável quando o paciente apresenta dificuldade na abertura da boca ${ }^{1,9}$.

Quanto ao uso da solução anestésica, a escolha por lidocaína $2 \%$ com epinefrina 1:100.000 é a mais comum na prática clínica, tida como padrão-ouro na literatura ${ }^{10}$. Outras opções ainda são usadas atualmente, a exemplo da articaína 4\% com epinefrina
1:100.000, cujo uso data desde 1999, com demonstrações em estudo de sua segurança e efetividade ${ }^{11-13}$.

A articaína é classificada como uma amida, que contém um anel de tiofeno e não de benzeno, fato incomum em outros anestésicos do tipo amida. O componente tiofeno aumenta sua lipossolubilidade, o que permite uma penetração mais eficaz nos tecidos ${ }^{2}$. Outra diferença molecular entre a articaína e os demais anestésicos locais do tipo amida é uma ligação extra tipo éster incorporada em sua molécula. Isto resulta na hidrólise da articaína pelas esterases plasmáticas. Sua hepatotoxicidade é diminuída por este motivo, tendo em vista que cerca de $90 \%$ de sua composição é metabolizada no sangue, enquanto que $10 \%$ são metabolizados no fígado ${ }^{14,15}$.

Apesar de a articaína ter uma melhor propriedade de difusão ${ }^{16}$, estudos prévios mostram que não há diferença significante quando se compara o uso da articaína $4 \%$ e da lidocaína $2 \%$ em bloqueio do nervo alveolar inferior ${ }^{11,17-19}$. Em contrapartida, outros estudos concluíram que a articaína 4\% com epinefrina 1:100.000 foi mais efetiva que lidocaína $2 \%$ com epinefrina 1:100.000 na infiltração bucal de molares inferiores ${ }^{20-22}$.

A falha na técnica anestésica normalmente é observada durante a fase crítica da exodontia, a odontossecção. O BNAI, mesmo quando aplicado corretamente, nem sempre resulta em anestesia pulpar ${ }^{23,24}$ e é durante esse momento da cirurgia que os tecidos pulpares são intensamente danificados, o que leva à percepção dolorosa do paciente. Na tentativa de diminuir a incidência de falhas e evitar o desconforto do paciente, vêm-se buscando alternativas, como infiltrações do nervo bucal ou bucal e lingual, com articaína $4 \%$ em molares inferiores na Endodontia $^{25}$. Apesar de a articaína se mostrar mais efetiva para a técnica infiltrativa, como dito anteriormente, outros estudos não obtiveram mais efetividade do que a lidocaína para BNAI, além de ter mostrado risco aumentado para parestesia ${ }^{26-28}$. Partindo-se desse ponto, o presente estudo avaliou e comparou a eficácia do BNAI pela técnica convencional e pela técnica de Vazirani-Akinosi, ambas com um tubete $(1,8 \mathrm{~mL})$ de lidocaína $2 \%$ com epinefrina 1:100.000 associado ao bloqueio do nervo bucal com meio tubete $(0,9 \mathrm{~mL})$ de articaína $4 \%$ com epinefrina 1:100.000, em cirurgias de extração de terceiros molares inferiores impactados.

\section{MATERIAL E MÉTODO}

\section{Amostra}

O presente estudo foi aprovado pelo Comitê de Ética em Pesquisa da Universidade Federal de Sergipe (CAAE: 12379613.9.0000.5546). Foram selecionados 160 pacientes voluntários do Departamento de Odontologia da Universidade Federal de Sergipe, após diagnóstico e indicação para exodontia do terceiro molar mandibular incluso e assintomático, com indicação para odontossecção, de acordo com a classificação de Pell, Gregori ${ }^{29}$. Destes, 80 pacientes foram submetidos ao BNAI pela técnica convencional, associado ao bloqueio do nervo 
bucal, e os outros 80 foram submetidos ao BNAI pela técnica de Vazirani-Akinosi, também associado ao bloqueio do nervo bucal.

Foram considerados critérios de exclusão: (I) pacientes com menos de 18 anos de idade; (II) qualquer problema de saúde geral, com base na história médica e no exame físico; (III) história de uso de qualquer tipo de medicamento nos 15 dias que antecederam o início da pesquisa; (IV) história de hipersensibilidade às drogas, substâncias ou materiais empregados neste experimento; (V) gravidez ou lactação; (VI) história prévia de pericoronarite.

\section{Antissepsia e Anestesia Local}

A antissepsia intraoral foi realizada por meio de um bochecho vigoroso, durante um minuto, com uma solução aquosa de digluconato de clorexidina a $0,12 \%$. Na antissepsia extraoral, utilizou-se uma solução alcoólica de polivinilpirrolidona-iodada (PVP-I) a $10 \%$.

Na técnica do BNAI convencional, o paciente foi posicionado em decúbito dorsal, com a boca bem aberta para permitir maior visibilidade e acesso ao local de injeção. Usou-se uma agulha longa de calibre 25. O ponto de introdução da agulha situou-se a três quartos da distância anteroposterior da margem anterior do ramo da mandíbula, até a parte mais profunda da rafe pterigomandibular, no ponto em que a rafe se curva para cima em direção ao palato. A agulha foi lentamente avançada até ser encontrada resistência óssea, numa profundidade média de 20 a 25 milímetros. A área alvo foi o nervo alveolar inferior, no ponto em que desce em direção ao forame da mandíbula, mas antes de penetrar no forame. O bisel estava voltado para o osso e a seringa estava apoiada nos pré-molares do lado oposto àquele que foi anestesiado. Após isso, fez-se a aspiração; quando negativo, foi feita a injeção do medicamento, na quantidade de um tubete $(1,8 \mathrm{~mL})$ de lidocaína a $2 \%$, com epinefrina 1:100.000. Nos casos contrários, o tubete foi trocado e a posição foi alterada até que a aspiração fosse negativa, sendo esta registrada. Associado a isso, posteriormente, foi feito o bloqueio do nervo bucal, injetando-se meio tubete $(0,9 \mathrm{~mL})$ de articaína a $4 \%$, com epinefrina 1:100.000, na mucosa distal e vestibular em relação ao terceiro molar.

Na técnica do BNAI de Vazirani-Akinosi, o paciente estava sentado e com a cabeça semirreclinada, com pescoço e ombros apoiados. A boca estava parcialmente fechada, sem contato oclusal, permitindo o relaxamento dos músculos da face para facilitar a melhor visualização da área. A área de inserção da agulha estava localizada entre o ramo da mandíbula e o tuber da maxila, na altura da junção mucogengival adjacente ao segundo ou terceiro molar superior. Com o dedo indicador ou polegar, afastou-se a bochecha lateralmente, para permitir uma melhor visualização da área, colocando a seringa paralela ao plano oclusal superior. Nesta posição, a agulha penetrou no tecido mole numa profundidade de $25 \mathrm{~mm}$ a partir do tuber da maxila. A solução de anestésico - sendo este o mesmo usado na técnica convencional do BNAI - foi depositada sobre o espaço medial pterigomandibular perto do ramo principal do nervo mandibular. Verificada a aspiração negativa, depositou-se um tubete $(1,8 \mathrm{~mL})$ de solução anestésica por aproximadamente 60 segundos, sendo removida a agulha em seguida. Após isso, foi realizado o bloqueio do nervo bucal, como descrito anteriormente.

\section{Procedimento Cirúrgico Propriamente Dito}

O procedimento cirúrgico ocorreu em uma sessão. A exodontia do terceiro molar inferior incluso foi realizada pelo operador, um Cirurgião Bucomaxilofacial do Departamento de Odontologia (DOD/UFS). A técnica cirúrgica empregada para todos os casos pode ser assim resumida: após a incisão de AVELLANAL $^{30}$ com lâmina de bisturi n. ${ }^{\circ} 15$ e o afastamento dos tecidos moles para exposição da loja cirúrgica, foram realizadas a ostectomia e a odontossecção por meio de brocas montadas em caneta de alta rotação, a $350.000 \mathrm{rpm}$, sob irrigação constante com solução de cloreto de sódio a $0,9 \%$. Em seguida, a exodontia foi realizada com o auxílio de elevadores retos do tipo Seldin, curetagem cuidadosa, regularização óssea e limpeza da loja cirúrgica por meio da irrigação abundante com soro fisiológico. Por fim, a sutura foi feita com pontos interrompidos.

Durante todo o procedimento cirúrgico, o paciente foi instruído a verbalizar quando do aparecimento de dor. Caso isso ocorresse, verificava-se a ineficiência da anestesia, sendo necessária então a aplicação de uma técnica anestésica complementar. Por conseguinte, registrava-se a ineficácia e o tempo cirúrgico em que ocorreu (incisão, diérese, odontossecção, luxação e sutura).

Os pacientes receberam orientação quanto aos cuidados relativos às medidas hemostáticas locais, alimentação, higienização da região operada e restrição de esforço físico, além de outras recomendações de rotina indicadas neste tipo de intervenção.

\section{MÉTODOS DE ESTUDO}

\section{Tratamento Estatístico dos Dados}

Os dados coletados e tabulados foram enviados ao Departamento de Estatística da UFS para realização dos testes estatísticos: teste do Qui-quadrado, teste de Mann-Whitney e teste exato de Fisher, com índice de significância de 5\%.

\section{RESULTADO}

A análise dos dados revelou que houve diferença significativa na quantidade de voluntários do gênero masculino $(\mathrm{p}=0,0004)$, como exposto na Tabela 1, observando o grupo submetido à técnica de Vazirani-Akinosi (Grupo 1), em relação àquele submetido à técnica convencional (Grupo 2). Além disso, os voluntários do Grupo 1 apresentaram maior idade, com média de 23,7 anos e Desvio Padrão (DP) de $\pm 4,7$ ( $p=0,0042$ ), em comparação com aqueles do Grupo 2, que obteve média de 21,5 anos e DP $\pm 3,1$. O significado desses achados e as influências destes nos resultados obtidos são discutíveis.

Considerando-se a aspiração positiva, foram avaliadas somente as técnicas de bloqueio do nervo alveolar inferior, 
não havendo diferença entre o Grupo 1 e o Grupo 2. Quanto à necessidade de complementação, não houve diferenças estatisticamente significantes ( $p=0,2453$ ) entre os dois grupos; também, em ambas as técnicas, obteve-se uma eficácia de $90 \%$ (Figura 1).

O momento em que ocorreu a dor também não mostrou diferenças estatisticamente significantes, sendo que, no Grupo 1, houve um episódio durante a incisão e sete durante a odontossecção, enquanto que, no Grupo 2, houve um caso durante a osteotomia e sete casos durante a odontossecção.

\section{DISCUSSÃO}

$\mathrm{Na}$ literatura, a quantidade de aspirações positivas da técnica convencional varia de $3,1 \%{ }^{6}$ a $20 \%{ }^{31}$. Já na técnica de Vazirani-Akinosi, Martínez-González et al. ${ }^{8}$ encontram $4 \%$ de aspirações positivas. Neste estudo, obteve-se um resultado abaixo dos apresentados na literatura tanto para a técnica convencional

Tabela 1. Distribuição relativa dos voluntários dentro dos dois grupos estudados

\begin{tabular}{cccc}
\hline Gênero & Grupo 1 & Grupo 2 & Total Geral \\
\hline Feminino & $65(81,3 \%)$ & $43(53,8 \%)$ & $108(67,5 \%)$ \\
Masculino & $15(18,8 \%)$ & $37(46,3 \%)$ & $52(32,5 \%)$ \\
Total Geral & $80(100 \%)$ & $80(100 \%)$ & $160(100 \%)$ \\
\hline
\end{tabular}

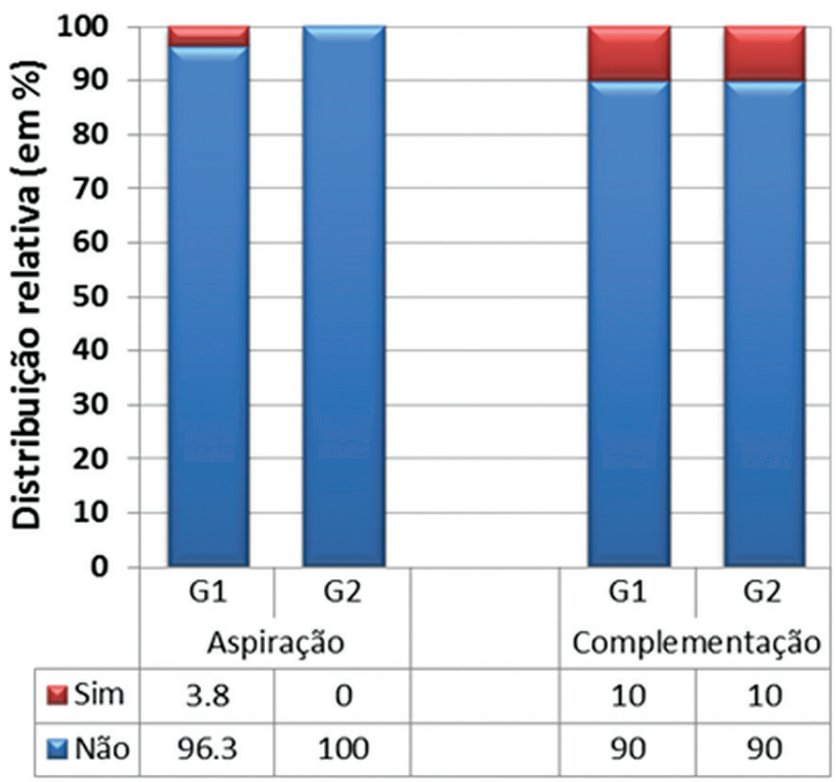

Figura 1. Distribuição relativa da aspiração positiva e da necessidade de complementação. quanto para a Vazirani-Akinosi, o que pode ser explicado pela variação da técnica individual do operador ${ }^{32}$.

Ao observar-se a eficácia das técnicas, percebe-se que não houve diferença entre as mesmas (ambas 90\%); adicionalmente, houve maior eficácia comparando-se aos resultados observados na literatura ${ }^{1,19,20,23}$. Isto pode ser explicado pelo uso associado da articaína no bloqueio do nervo bucal, o que não é, ainda, considerado uma prática clínica de rotina. No entanto, observa-se, na literatura atual, que as taxas de eficácia do BNAI variam de $80 \%$ a $85 \%^{2}$, sendo menores às obtidas neste estudo.

Estudos comparativos entre lidocaína e articaína demonstram que não há diferença significante no sucesso da anestesia pulpar em caninos maxilares, bem como durante o bloqueio do nervo alveolar inferior ${ }^{10,17-19,33}$. No presente estudo, optou-se pelo uso da lidocaína 2\% com epinefrina 1:100.000 no bloqueio do nervo alveolar inferior, pois, como citado anteriormente, não havia diferença entre os dois anestésicos para tal finalidade; adicionalmente, a lidocaína 2\% com epinefrina 1:100.000 é mais acessível do ponto de vista prático e possui uma concentração menor de sal anestésico (2\%) em relação à articaína (4\%). Todavia, observou-se que a articaína foi mais efetiva na anestesia pulpar de molares inferiores, através da técnica de infiltração bucal, do que a lidocaína ${ }^{20-22}$, o que levou a optar pelo seu uso (articaína $4 \%$ com epinefrina 1:100.000) no bloqueio do nervo bucal. Cabe aqui ressaltar: quanto à associação de anestésicos utilizada, foram usadas quantidades muito abaixo da dose máxima recomendada pelos fabricantes, tendo como parâmetro a droga que tem menor dose máxima ${ }^{2}$.

Em relação ao momento cirúrgico em que ocorreu a dor, verificou-se que a maioria ocorreu durante a odontossecção. É sabido que a polpa é um local de dificuldade para anestesia e que o BNAI, mesmo aplicado corretamente, em algumas circunstâncias, não resulta em anestesia pulpar ${ }^{23,24}$. $\mathrm{Na}$ odontossecção, como a polpa será diretamente lesada, torna esse um momento cirúrgico crítico para o aparecimento da dor.

\section{CONCLUSÃO}

Conclui-se, assim, que não houve diferença quanto a eficácia e aspiração positiva, comparando-se as técnicas BNAI convencional e BNAI de Vazirani-Akinosi. Sendo assim, ambas as técnicas são viáveis para a prática clínica de rotina.

O uso da articaína 4\% com epinefrina 1:100.000 no bloqueio do nervo bucal após o bloqueio do nervo alveolar inferior possivelmente aumentou a eficácia de ambas as técnicas quando comparada aos dados de eficácia da literatura.

Sugere-se a repetição deste protocolo, associando a um grupo controle no qual o mesmo anestésico usado no bloqueio do nervo alveolar inferior fosse o mesmo para o nervo bucal. 


\section{REFERÊNCIAS}

1. Goldberg S, Reader A, Drum M, Nusstein J, Beck M. Comparison of the anesthetic efficacy of the conventional inferior alveolar, Gow-Gates, and Vazirani-Akinosi techniques. J Endod. 2008; 34:1306-11. http://dx.doi.org/10.1016/j.joen.2008.07.025

2. Malamed SF. Handbook of local anesthesia. 5th ed. St. Louis: Mosby; 2004.

3. Hargreaves KM, Keiser K. Local anesthetic failure in endodontics: mechanisms and management. Endod Topics. 2002; 1:26-39. http:// dx.doi.org/10.1034/j.1601-1546.2002.10103.x

4. Kuster CG, Udin RD. Frequency of accidental intravascular injection of local anesthetics in children. J Dent Child. 1985 May-Jun; 52(3):183-7.

5. Harris SC. Aspiration before injection of dental local anesthetics. J Oral Surg. 1957; 15:299-303.

6. Taghavi Zenouz A, Ebrahimi H, Mahdipour M, Pourshahidi S, Amini P, Vatankhah M. The incidence of intravascular needle entrance during inferior alveolar nerve block injection. J Dent Res Dent Clin Dent Prospects. 2008 Winter; 2(1):38-41. doi: 10.5681/joddd.2008.008. Epub 2008 May 15.

7. Vasconcelos BCE, Freitas KCM, Almeida RAC, Mauricio HA. A importância da técnica de aspiração prévia ao bloqueio anestésico do nervo alveolar inferior. Rev Cir Traumatol Buco-Maxilo-Fac. 2007; 7:29-36.

8. Martínez-González JM, Peña BB, Cáliz FF, Marín LSH, DiagoMP. Estudio comparativo entre el bloqueo mandibular directo y la técnica de Akinosi. Med Oral. 2003; 8:143-9.

9. Sisk AL. Evaluation of the akinosi mandibular block technique in oral surgery. J Oral Maxillofac Surg. 1986; 44:113-5. http://dx.doi.org/ 10.1016/0278-2391(86)90192-8

10. Rowson JE, Preshaw PM. The use of lignocaine in dental practice: results of a survey of a group of general and hospital dental practitioners. J Dent. 1997; 25:431-3. http://dx.doi.org/10.1016/S0300-5712(96)00062-0

11. Malamed SF, Gagnon S, Leblanc D. Efficacy of articaine: a new amide local anesthetic. J Am Dent Assoc. 2000; 131:635-42. http://dx.doi. org/10.14219/jada.archive.2000.0237

12. Malamed SF, Gagnon S, LeBlanc D. Articaine hydrochloride: a study of the safety of a new amide local anesthetic. J Am Dent Assoc. 2001; 132:177-85. http://dx.doi.org/10.14219/jada.archive.2001.0152

13. Hersh EV, Giannakopoulos H, Levin LM, Secreto S, Moore PA, Peterson C, et al. The pharmacokinetics and cardiovascular effects of high-dose articaine with 1:100,000 and 1:200,000 epinephrine. J Am Dent Assoc. 2006 Nov; 137(11):1562-71. http://dx.doi.org/10.14219/ jada.archive.2006.0092

14. Srinivasan N, Kavitha M, Loganathan CS, Padmini G. Comparison of anesthetic efficacy of $4 \%$ articaine and $2 \%$ lidocaine for maxillary buccal infiltration in patients with irreversible pulpitis. Oral Surg Oral Med Oral Pathol Oral Radiol Endod. 2009; 107:133-6. http:// dx.doi.org/10.1016/j.tripleo.2008.09.002

15. Isen DA. Articaine: pharmacology and clinical use of a recently approved local anesthetic. Dent Today. 2000; 19:72-7.

16. Oertel R, Rahn R, Kirch W. Clinical pharmacokinetics of articaine. Clin Pharmacokinet. 1997; 33:417-25. http://dx.doi.org/10.2165/000 03088-199733060-00002

17. Tortamano IP, Siviero M, Costa CG, Buscariolo IA, Armonia PL. A comparison of the anesthetic efficacy of articaine and lidocaine in patients with irreversible pulpitis. J Endod. 2009; 35:165-8. http://dx.doi.org/10.1016/j.joen.2008.10.020

18. Claffey E, Reader A, Nusstein J, Beck M, Weaver J. Anesthetic efficacy of articaine for inferior alveolar nerve blocks in patients with irreversible pulpitis. J Endod. 2004 Aug; 30(8):568-71. http://dx.doi.org/10.1097/01.DON.0000125317.21892.8F

19. Mikesell P, Nusstein J, Reader A, Beck M, Weaver J. A comparison of articaine and lidocaine for inferior alveolar nerve blocks. J Endod. 2005 Apr; 31(4):265-70. http://dx.doi.org/10.1097/01.don.0000140576.36513.cb

20. Kanaa MD, Whitworth JM, Corbett IP, Meechan JG. Articaine and lidocaine mandibular buccal infiltration anesthesia: a prospective randomized double-blind crossover study. J Endod. 2006; 32:296-8. http://dx.doi.org/10.1016/j.joen.2005.09.016

21. Robertson D, Nusstein J, Reader A, Beck M, McCartney M. The anesthetic efficacy of articaine in buccal infiltration of mandibular posterior teeth. J Am Dent Assoc. 2007 Aug; 138(8):1104-12. http://dx.doi.org/10.14219/jada.archive.2007.0324

22. Jung IY, Kim JH, Kim ES, Lee CY, Lee SJ. An evaluation of buccal infiltrations and inferior alveolar nerve blocks in pulpal anesthesia for mandibular first molars. J Endod. 2008 Jan; 34(1):11-3. http://dx.doi.org/10.1016/j.joen.2007.09.006

23. Nusstein J, Reacher A, Beck M. Anesthetic efficacy different volumes of lidocaine with epinephrine for inferior alveolar nerve blocks. Gen Dent. 2002; 50:372-5, quiz 376-7.

24. Lai TN, Lin CP, Kok SH, Yang PJ, Kuo YS, Lan WH, et al. Evaluation of mandibular block using a standardized method. Oral Surg Oral Med Oral Pathol Oral Radiol Endod. 2006 Oct; 102(4):462-8. Epub 2006 Jun 8. http://dx.doi.org/10.1016/j.tripleo.2005.12.003

25. Aggarwal V, Singla M, Kabi D. Comparative evaluation of anesthetic efficacy of Gow-Gates mandibular conduction anesthesia, Vazirani-Akinosi technique, buccal-plus-lingual infiltrations, and conventional inferior alveolar nerve anesthesia in patients with irreversible pulpitis. Oral Surg Oral Med Oral Pathol Oral Radiol Endod. 2010 Feb; 109(2):303-8. http://dx.doi.org/10.1016/j. tripleo.2009.09.016

26. van Eden SP, Patel MF. Prolonged paraesthesia following inferior alveolar nerve block using articaine. Br J Oral Maxillofac Surg 2002 Dec; 40(6):519-20. http://dx.doi.org/10.1016/S0266435602002231 
27. Garisto GA, Gaffen AS, Lawrence HP, Tenenbaum HC, Haas DA. Occurrence of paresthesia after dental local anesthetic administration in the United States. J Am Dent Assoc. 2010; 41:836-44. http://dx.doi.org/10.14219/jada.archive.2010.0281

28. Pogrel MA. Permanent nerve damage from inferior alveolar nerve blocks: a current update. J Calif Dent Assoc. 2012; 40:795-7.

29. Pell GJ, Gregory BT. Impacted mandibular third molars: classification and modified techniques for removal. Dent Digest. 1933; 39: 330-8.

30. Avellanal CD. Tratado de odontologia: cirurgia odontomaxilar. Buenos Aires: Editora Ediar; 1946.

31. Frangiskos F, Stavrou E, Merenditis N, Tsitsogianis H, Vardas E, Antonopoulou I. Incidence of penetration of a blood vessel during inferior alveolar nerve block. Br J Oral Maxillofac Surg. 2003 Jun; 41(3):188-9. http://dx.doi.org/10.1016/S0266-4356(03)00045-7

32. Meechan JG, Blair GS. Clinical experience in oral surgery with 2 different automatic aspirating syringes. Int J Oral Maxillofac Surg. 1989 Apr; 18(2):87-9. http://dx.doi.org/10.1016/S0901-5027(89)80137-7

33. Corbett IP, Kanaa MD, Whitworth JM, Meechan JG. Articaine infiltration for anesthesia of mandibular first molars. J Endod. 2008 May;34(5):514-8. http://dx.doi.org/10.1016/j.joen.2008.02.042

\section{CONFLITOS DE INTERESSE}

Os autores declaram não haver conflitos de interesse.

\section{AUTOR PARA CORRESPONDÊNCIA}

Danilo de Paula Ribeiro Borges

Av. Beira-Mar, 580, apto. 401, Treze de Julho, 49020-010 Aracaju - SE, Brasil

e-mail: daniloprb@gmail.com

Recebido: Junho 6, 2013

Aprovado: Novembro 13, 2013 